Journal of Computer Science 8 (3): 420-424, 2012

ISSN 1549-3636

(C) 2012 Science Publications

\title{
A Cross Layer Frame Work for Enhanced Quality of Service Provisioning in Worldwide Interoperability for Microwave Access Networks
}

\author{
${ }^{1}$ Jagadeesh Kumar, S.J.K. and ${ }^{2}$ T. Purusothaman \\ ${ }^{1}$ Department of CSE, \\ V.L.B. Janakiammal College of Engineering and Technology, Coimbatore, India \\ ${ }^{2}$ Department of CSE/IT, Government College of Technology, Coimbatore, 13, India
}

\begin{abstract}
Problem statement: The demand for multimedia applications in WiMAX networks is growing at a rapid pace. A method for guaranteeing Quality of Service (QoS) for different classes of traffic is therefore gaining importance. Hence designing and analyzing multimedia traffic and QoS parameters has become central to this problem. In this study, we propose a cross layer frame work in which a coordination between the Medium Access Control layer (MAC) and the Physical layer (PHY) is established for guaranteeing the QoS requirement in a multiclass traffic WiMAX environment. Two traffic classes' real time Polling Service (rtPS) and non real time Polling Service (nrtPS) are considered for analyzing the performance. Approach: The objective of study is to guarantee QoS for multiple service class traffic in a multiple connection environment in WiMAX network. A cross layer design approach is used for this purpose. A priority scheduler at the MAC layer schedules the traffic based on channel state information. The Adaptive modulation and coding scheme is used at the physical layer that adapts to the scheduled traffic to stabilize the QoS requirements of different traffic classes. Results: The Priority value is estimated using the Friis equation that calculates the received power and determines the SNR. The average throughput, average bytes received and the packet loss are plotted against time. This indicated that these QoS parameters are stable over a period of time. Conclusion: A cross layer frame work was developed based on a scheduler for QoS stability that uses CSI at the MAC layer and an AMC at the PHY layer in WiMax Networks. The scheduler enjoyed flexibility and scalability, whose performance was evaluated against existing systems through simulation.
\end{abstract}

Key words: Cross layer design, QoS provisioning, Quality of Service (QoS), real time Polling Service (rtPS), non real time Polling Service (nrtPS), Medium Access Control (MAC), CrossLayer Design (CLD), Subscriber Stations (SSs), Customer Premises Equipment (CPE)

\section{INTRODUCTION}

Providing QoS-guaranteed services is necessary for next generation wireless networks, including IEEE 802.16 standard based networks. Such networks are envisioned to support multimedia services with different QoS requirements for different applications including voice, data and real time, or streaming video/audio. However, the aforementioned standards define only QoS architecture and signaling, but do not specify the scheduling algorithm that will ultimately provide QoS support.

Scheduling for QoS: Scheduling plays an important role in QoS provision. Although many traffic scheduling algorithms are available for wire-line networks, they cannot be directly applied to wireless networks because of the fundamental differences between the two. For example, traditional schedulers for wire-line networks only consider traffic and queuing status; however, channel capacity in wireless networks is time varying due to multipath fading and Doppler effects. Even if large bandwidth is allocated to a certain connection, the prescribed delay or throughput performance may not be satisfied and the allocated bandwidth is wasted when the wireless channel experiences deep fades (Liu et al., 2006).

Cross layer design: In the past, layering has lead to the rapid development of interoperable systems, but limited the performance of the overall architecture, due to the lack of coordination among layers (Kliazovich et al., 2008). This has necessitated the introduction of Cross-Layer Design (CLD) solutions which would make it more

Corresponding Author: Jagadeesh Kumar, S.J.K., Department of CSE, V.L.B. Janakiammal College of Engineering and Technology, Coimbatore, India 
suitable for operation in the modern heterogeneous wireless environment. CLD allows communication to take place even between nonadjacent layers through additional entities introduced into the system's architecture. However, there is no reference model that specifies the functionality each new entity (i.e., module) must realize in a cross-layer design solution (Foukalas et al., 2008).

Proposed architecture: The proposed scheme defines a cross layer frame work in which the MAC and PHY layers coordinate between them to guarantee the QoS for a multi class traffic in a multi channel environment. We propose channel state information based scheduling in the MAC layer for each type of connection which then schedules on AMC based slots in the PHY layer that adapts to higher layer QoS requirements, service flow's types and queuing state information. Based on the scheduling mechanism combined with adaptive modulation scheme, a fair and efficient QoS guarantees in terms of maximum delay requirement for real-time SFs and minimum reserved data rate for non real-time SFs flows are achieved (Ali-Yahiya et al., 2009).

The article's organization is as follow. First an overview of the WiMAX architecture is provided. This is followed by the details of the proposed architecture. A detailed performance analysis of our scheme follows. The study ends with conclusion and future work.

WiMAX architecture: Broadband wireless architecture is being standardized by the IEEE 802.16 Working Group (WG) and the Worldwide Interoperability for Microwave Access (WiMAX) forum. The basic IEEE 802.16 architecture consists of one Base Station (BS) and one or more Subscriber Stations (SSs) (Liu et al., 2006; Geetha and Jayaparvathy, 2011). Figure 1 shows a typical IEEE 802.16 network in PMP mode comprising a Base Station (BS) that communicates with one or more Subscriber Stations (SS) known as Customer Premises Equipment (CPE). IEEE 802.16 specifies the following modes of deployment architectures.

Point-To-Point (PTP): A connection between one BS and one SS. The PTP mode extends the range over the PMP mode.

Point-to-Multi Point (PMP): A connection between one BS and multiple SS nodes.

The BS always coordinates the uplink and downlink transmission. This mode supports multicast communication.
Point-To-Consecutive Point (PTCM): It involves the creation of a closed loop through multiple PTP connections.

Mesh: SSs can communicate with each other without the coordination of a BS.

Both BS and SS are stationary while clients connected to SS can be mobile. BS acts as a central entity to transfer all the data from SSs in PMP architecture. Two or more SSs are not allowed to communicate directly. Transmissions take place through two independent channels-downlink channels (from BS to SS) and uplink channel (from SS to BS). The uplink channel is shared among all the SSs while the downlink channel is used only by BS.

Cross layer mechanism: The proposed architecture is a generic architecture for providing QoS guarantees in IEEE 802.16-compliant networks. The WiMAX network operates in a PMP Mode. The Cross Layer mechanism is implemented by the scheduler who uses the available CSI at the MAC layer and QSI from the application layer. At every timeslot, the scheduling algorithm has to produce rate allocation and power for all the $\mathrm{k}$ users, which is based on the observation of the current Channel State Information (CSI) from the physical layer and the Queue State Information (QSI) from the application layer. Rate allocation and power allocation are selected so that they optimize some system objectives.

Design of MAC scheduler: In this study, a prioritybased scheduler shown in Fig. 2, is proposed at the MAC layer for multiple connections with diverse QoS requirements, where each connection employs Adaptive Modulation and Coding (AMC) scheme at the Physical (PHY) layer. A priority value is estimated for each connection admitted in the system and is updated dynamically depending on the CSI, QSI and service priority across layers. Thus, the connection with the highest priority is scheduled each time. The scheduler provides prescribed QoS guarantees and utilizes the bandwidth efficiently while enjoying low implementation complexity, flexibility and scalability. Multiple Subscriber Stations (SS) are connected to the Base Station (BS) over wireless channels, where multiple connections (sessions, flows) can be supported by each SS.

Estimation of CSI and QSI: The Channel State Information consists of the physical layer constraints such as Channel fading, Multi-path propagation, Reflection, Scattering and other climatic effects on the channel. The CSI is estimated based on the signal strength and Signal to Noise Ratio (SNR) at the receiver. Based on 
the CSI, Adaptive Modulation and Coding is done at the transmitter to reduce Packet Error Rate. The Signal Strength is estimated by the Friis Eq. 1 given by:

$$
\operatorname{Pr}=\frac{\mathrm{Pt} * \mathrm{Gt} * \mathrm{Gr} * \mathrm{Ht} * \mathrm{Hr} * \lambda^{2}}{(4 * \lambda * \mathrm{~d})^{2} * \mathrm{~L}} \text { Watts }
$$

Where:

$$
\begin{aligned}
& \mathrm{Pt}=\text { Transmitted power } \\
& \text { Gt and } \mathrm{Gr}=\text { Gain of transmitter and receiver }
\end{aligned}
$$

$\mathrm{SNR}=\log _{10}(\mathrm{Pt})-\log _{10}(\mathrm{Pr}) \mathrm{dB}$

The priority value is calculated based on the Channel State Information. A dequeue is made from the highest priority queue and also the priority is dynamically updated with time. Least priority is given to connections with poor CSI thus preventing high packet loss and packet error rate.

QSI regarding the type of service is taken from each packet and classified according to the priority:

\section{IFQ_RTPROTO \\ IFQ_REALTIME \\ IFQ_LOWDELAY \\ IFQ_NORMAL}

AMC mode at the PHY layer: At the physical layer, we assume that multiple transmission modes are available, with each mode consisting of a specific modulation and FEC code pair.

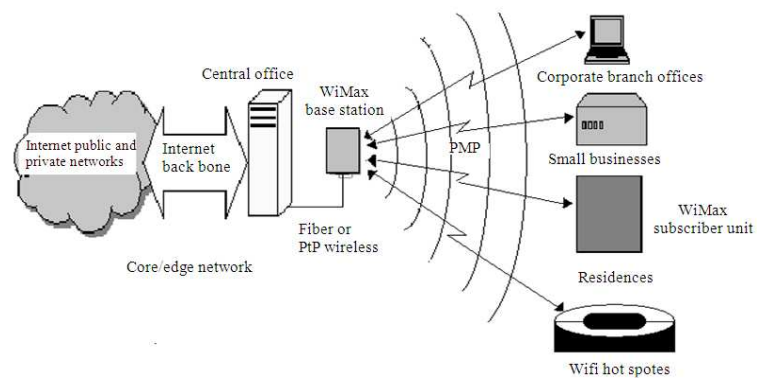

Fig. 1: IEEE 802.16 PMP mode architecture
Based on the acquired CSI as listed in Table 2, the AMC selector determines the modulation-coding pair (mode). The AMC controller then updates the transmission mode at the transmitter. Coherent demodulation and Maximum-Likelihood (ML) decoding are used at the receiver (Liu et al., 2004).

We consider the following group of transmission modes as in the IEEE 802.16 standard. Transmission Modes (TM): The modulations are $M_{n}$-ary rectangular/square Quadrature Amplitude Modulators (QAMs) and the FEC codes are Reed-Solomon (RS) concatenated with Convolution Codes (CC). Although we focus on this TM, other transmission modes can be similarly constructed.

Based on the SNR value and Signal Strength, a Threshold value is fixed and utilized for Modulation Adaptation. Here in our work QAM 64 Modulation is used for good channel conditions where the SNR is less than $25 \mathrm{~dB}$ and QAM 16 is used for average Channel conditions when SNR is greater than $25 \mathrm{~dB}$. A low level modulation is used that reduces the Packet Error Rate and Loss Rate.

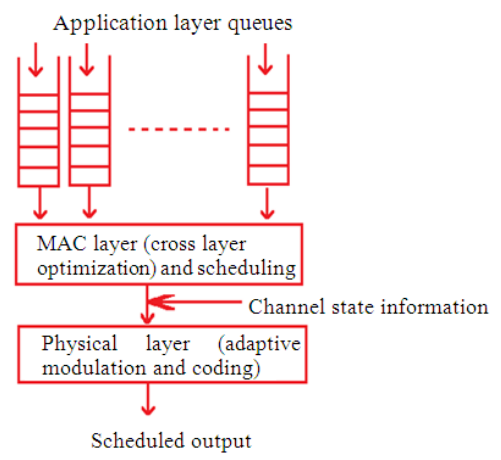

Fig. 2: System design for the cross layer mechanism

Table 1: Simulation parameters

\begin{tabular}{ll}
\hline Parameter & Value \\
\hline Burst time & $500 \mathrm{~ms}$ for video packets \\
Idle time & $100 \mathrm{~ms}$ for telnet packets \\
& $10 \mathrm{~ms}$ for video packets \\
Maximum height of antenna & $500 \mathrm{~ms}$ for telnet packets \\
Average coverage area of base station & $1.5 \mathrm{~m}$ \\
Transmission power of base station & $500 \mathrm{~m}$ \\
Frequency & $0.025 \mathrm{~W}$ \\
Propagation & $914 \mathrm{MHz}$ \\
\hline
\end{tabular}

Table 2: Calculation of Channel State Information

\begin{tabular}{llll}
\hline Node no. & $\begin{array}{l}\text { Distance } \\
\text { from BS }\end{array}$ & $\begin{array}{l}\text { Received signal } \\
\text { Power (Pr) }\end{array}$ & $\begin{array}{l}\text { Signal to } \\
\text { Noise Ratio (SNR) }\end{array}$ \\
\hline 45 & 107.8758756 & $1.47875 \mathrm{e}-09$ & 16.875387 \\
46 & 278.3078730 & $1.67983 \mathrm{e}-09$ & 17.298369 \\
47 & 168.9838740 & $1.87532 \mathrm{e}-09$ & 18.398794 \\
48 & 148.9487620 & $1.56282 \mathrm{e}-09$ & 17.943876 \\
\hline
\end{tabular}




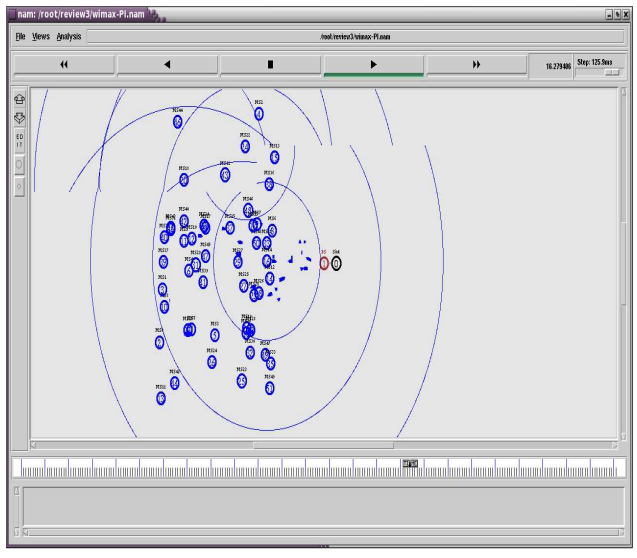

Fig. 3: Simulation of PI scheduler
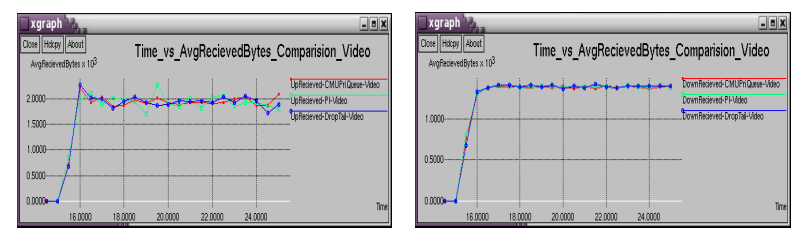

Fig. 4: Comparison of average bytes received for video traffic
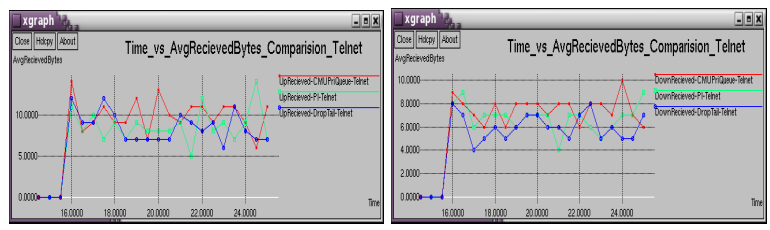

Fig. 5: Comparison of average bytes received for telnet traffic
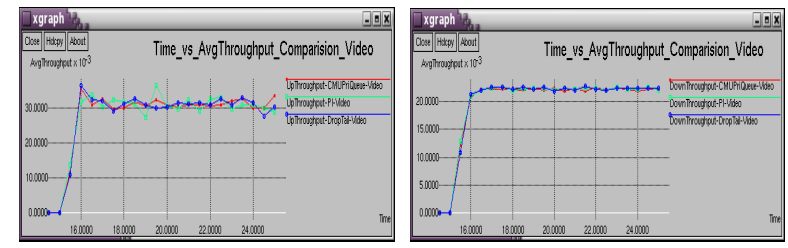

Fig. 6: Comparison of average throughput for video traffic

\section{Performance comparison:}

Simulation: Figure 3 shows the simulation of the PI scheduler. The WiMAX environment is simulated using NS 2.29 with 50 Mobile Nodes where 25 nodes exchanged Video Streaming Traffic and 25 nodes exchanged telnet traffic. The Scheduler Used is PI Scheduler and the mode of operation was Point-tomultipoint. The bandwidth is 10 Mbps. Following are the other parameters specified in Table 1 .
The Environment for our system is designed with the two different set of services, rtPS with streaming video and nrtPS with telnet traffic. Different set of queues are established for different services with different priorities. The priorities are calculated based on the following:

\section{$\mathrm{UGS}>\mathrm{rtPS}>\operatorname{nrtPS}>\mathrm{BE}$}

Allocation of slots for UGS services is given highest priority.A fixed number of slots are separately allocated for UGS services. The remaining slots are allocated to particular service among rtPS, nrtPS and $\mathrm{BE}$ according to priority.

Average bytes received: Figure 4. shows the comparison graphs for rtPS traffic in which the average bytes received increases with the times, The Uplink performance is better than downlink performance and also all the scheduling algorithms produce almost same performance.

From Figure 5, it is found that average bytes received in case of nrtPS traffic varies within a range of values as compared to rtPS traffic with increase in time. The Uplink performance is better than downlink performance and also all the scheduling algorithms produce almost same performance.

Average throughput: Figure 6 shows the comparison of average throughput for rtPS traffic. The variation in the throughput is more pronounced in uplink than downlink throughput.

Packet loss: Figure 7 shows the comparison graphs for packet loss considering video traffic. It is found that as the time increases the Packet Loss increases. The proposed Cross layer Scheduler produces very Minimum packet loss of 350 (Uplink) and 240 (Downlink) when compared to higher packet loss and Packet error rate of 400 (Uplink) and 600 (downlink) respectively in existing scheduling systems.

Thus the proposed scheme provides minimum Packet Error Rate Performance.

From the comparison graphs shown in Fig. 8 which considers telnet traffic, it was found that as the time increases the Packet Loss increases. The proposed Cross layer Scheduler produces very Minimum packet loss of 5 (Uplink) and 0 (Downlink) when compared to higher packet loss and Packet error rate of 10 (Uplink) and 1 (downlink) respectively in existing scheduling systems. Thus the proposed method provides minimum Packet Error Rate Performance. 

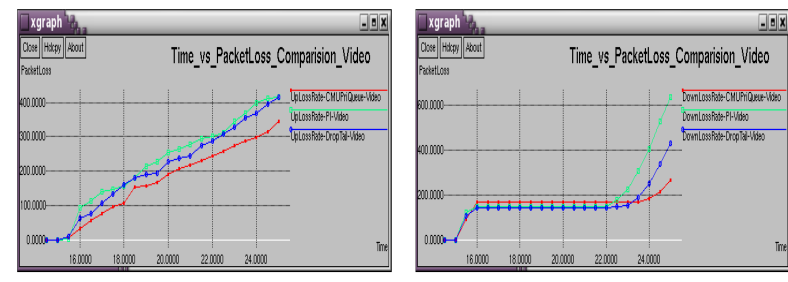

Fig. 7: Comparison of packet loss for video traffic
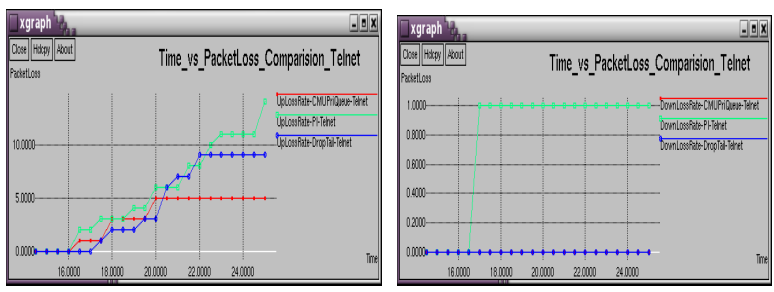

Fig. 8: Comparison of packet loss for telnet traffic

\section{MATERIALS AND METHODS}

Simulation was carried out using NS2 2.29. The wimax environment was created using 50 nodes out of which 25 nodes each were designed to handle video and telnet. The PI scheduling algorithm working at the MAC layer schedules the traffic using the estimated priority value. The slot allocation is done at the PHY layer using Adaptive modulation and coding. This provides an integrated QoS provisioning based on the cross layer frame work.

\section{RESULTS}

The graphs plotted for the average throughput, average bytes received and the packet loss for the two types of traffic show the QoS provisioning capability of the frame work.

\section{DISCUSSION}

The average bytes received, average throughput and packet loss observed from the model demonstrate that The variation in the throughput is more pronounced in uplink than downlink throughput. It is also found that as the time increases the Packet Loss increases. Also the average bytes received in case of nrtPS traffic varies within a range of values as compared to rtPS traffic with increase in time

\section{CONCLUSION}

A cross layer frame work has been developed based on a scheduler that uses CSI and QSI for QoS stability at the MAC layer and an AMC at the PHY layer in WiMax Networks. This system offered Minimum Packet Error rate, Delay and Rate guarantees for rt and nrt traffic. Furthermore our scheduler enjoyed flexibility and scalability, whose performance was evaluated against existing systems through simulation.

The effects of imperfect channel state information due to estimation error and feedback latency can be considered while extending study. Network and Application layer issues can also be considered for scheduling. A generic QoS control Architecture can be designed by combining different layers for specific applications based QoS requirements.

\section{REFERENCES}

Ali-Yahiya, T., A.L. Beylot and G. Pujolle, 2009. An adaptive cross-layer design for multiservice scheduling in OFDMA based mobile WiMAX systems. Comput. Commun., 32: 531-539. DOI: 10.1016/j.comcom.2008.09.016

Foukalas, F., V. Gazis and N. Alonistioti, 2008. Crosslayer design proposals for wireless mobile networks: A survey and taxonomy. IEEE Commun. Sur. Tutorials, 10: 70-85. DOI: 10.1109/COMST.2008.4483671

Jain, A. and A.K. Verma, 0000. Comparative study of Scheduling Algorithms for WiMAX. CSED Department, Thapar University, Patiyala.

Kliazovich, D., M. Devetsikiotis and F. Granelli, 2008. Formal Methods in Cross Layer Modeling And Optimization Of Wireless Networks. Handbook of Research on Heterogeneous Next Generation Networking: Innovations and Platforms, Kotsopoulos, S. and K.G. Ioannou (Eds). Idea Group Inc., Hershey, ISBN-10: 1605661082, pp: 1-24.

Liu, Q., S. Zhou and G.B. Giannakis, 2004. Cross-layer combining of adaptive modulation and coding with truncated ARQ over wireless links. IEEE Trans. Wireless Commun., 3: 1746-1755. DOI: 10.1109/TWC.2004.833474

Liu, Q., X. Wang and G.B. Giannakis, 2006. A Crosslayer scheduling algorithm with QoS support in wireless networks. IEEE Trans. Vech. Technol., 55: 839-847. DOI: 10.1109/TVT.2006.873832

So-In, C., R. Jain and A.K. Tamimi, 2009. Scheduling in IEEE 802.16e mobile WiMAX networks. Key issues and a survey. IEEE J. Selected Areas Commun., 27: 156-171. DOI: 10.1109/JSAC.2009.090207

Zou, J. and D. Zhao, 2007. Real-time CBR traffic scheduling in IEEE 802.16-based wireless mesh networks. Wireless Netw., 15: 65-72. DOI: 10.1007/s11276-007-0025-X 